\title{
Tooth Implant-supported Obturator for a Hemimaxillectomy Patient
}

\author{
${ }^{1}$ Chandan Rathod, ${ }^{2}$ Makrand Sapat, ${ }^{3}$ Fatema Sodawala, ${ }^{4}$ Javed Sodawala
}

\begin{abstract}
The esthetic and functional rehabilitation of the patients with palatal defects using obturator poses challenge to the clinician. The use of existing teeth along with implants helps in providing good retention, support, and stability to the prosthesis. Ball-socket type of attachments offer efficient, yet economical alternative to these patients. This case report describes management of a hemimaxillectomy adult patient with a class II palatal defect using tooth implant-supported obturator. Osseointegrated implants and direct overdenture post with ball-socket type of attachments were used to construct a definitive obturator for prosthetic rehabilitation of this patient.
\end{abstract}

Keywords: Hemimaxillectomy, Implant, Obturator.

How to cite this article: Rathod C, Sapat M, Sodawala F, Sodawala J. Tooth Implant-supported Obturator for a Hemimaxillectomy Patient. Int J Prosthodont Restor Dent 2017;7(4):129-132.

\section{Source of support: Nil}

Conflict of interest: None

\section{INTRODUCTION}

The Glossary of Prosthodontic Terms has defined obturator as "maxillofacial prostheses which are used to close a congenital or an acquired tissue opening, primarily of hard palate and or contiguous alveolar structures". ${ }^{1}$ The obturator must establish oronasal separation to prevent oral secretions and food from entering the nasal cavity during mastication and deglutition and nasal secretions from entering the oral cavity during respiration. Also, the oronasal relationships must be maintained for speech articulation and resonance.

The retention of the obturator in the oral cavity at the area of surgical defect is critical. ${ }^{2,3}$ Patients having surgical

\footnotetext{
${ }^{1,4}$ Reader, ${ }^{2,3}$ Postgraduate Student

${ }^{1-3}$ Department of Prosthodontics and Crown and Bridge, Rungta College of Dental Sciences \& Research, Bhilai, Chhattisgarh India

${ }^{4}$ Department of Orthodontics and Dentofacial Orthopedics Rungta College of Dental Sciences \& Research, Bhilai Chhattisgarh, India

Corresponding Author: Fatema Sodawala, Postgraduate Student, Department of Prosthodontics and Crown and Bridge, Rungta College of Dental Sciences \& Research Bhilai, Chhattisgarh, India, Phone: +918823063672 , e-mail: fatemaelectricwala23@gmail.com
}

defects with unilateral few remaining teeth face difficulty in retaining this prosthesis. ${ }^{4}$ In such cases, tooth implantsupported prosthesis will meet all esthetic and functional demands of the patients by bilateral stabilization of the prosthesis. This case report describes management of a hemimaxillectomy adult patient using a tooth implantsupported obturator.

\section{CASE REPORT}

A male patient aged 59 years reported to our department with a complaint of loose fitting obturator, retained with the extracoronal attachments in relation to maxillary right first premolar and left lateral. He gave a previous history of squamous cell carcinoma affecting left maxilla for which he had undergone left hemimaxillectomy 4 years back.

Maxillary arch was U-shaped with class II palatal defect $^{5}$ (Fig. 1A). Teeth present in the maxillary arch were right central, lateral, canine, and first premolar and left central and lateral, and porcelain fused to metal crowns were present on all the maxillary teeth (Fig. 1B). Grade III mobility in relation to maxillary left central and lateral was also present.

\section{Treatment Plan}

Patient was advised a tooth implant-supported obturator. Alternatively, extraction of all the maxillary teeth and a maxillary complete denture were also advised to the patient. Patient was not willing for extraction of all his maxillary teeth. Therefore, a tooth implant-supported obturator was opted for this patient.

\section{Treatment Progress}

Extraction of maxillary left central and lateral and root canal treatment of maxillary right canine and first premolar was done. Maxillary impression was made using irreversible hydrocolloid (Marieflex, Septodont Healthcare India Ltd., Panvel, India). Interim obturator with circumferential clasp on maxillary right canine and first premolar was constructed using heat cure acrylic (DPIHeat Cure, Dental Products of India Ltd., Mumbai, India) and delivered to the patient immediately (Fig. 2).

After 1 month, two implants-3.8 $\times 11$ and $3.8 \times 9.5 \mathrm{~mm}$ (Myriad Plus, Equinox Medical Technology, Amersfoort, 

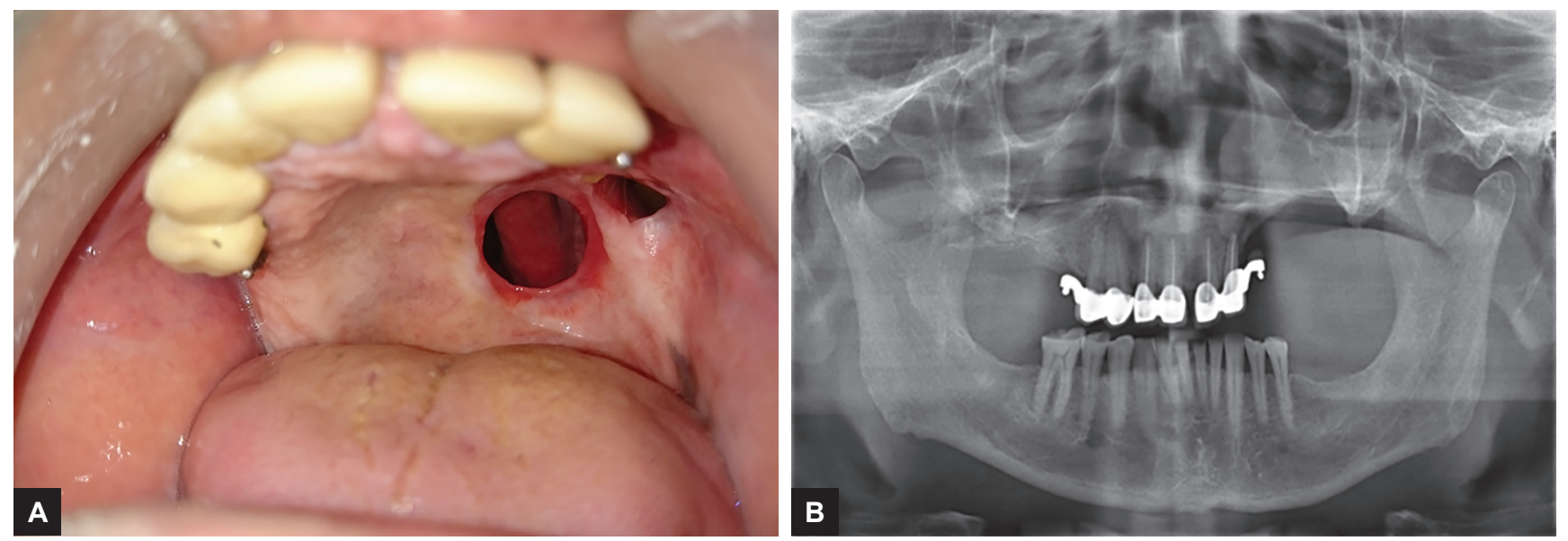

Figs 1A and B: Pretreatment. (A) Maxillary occlusal photograph showing class II palatal defect and (B) panoramic radiograph showing extracoronal attachments in relation to 14 and 22

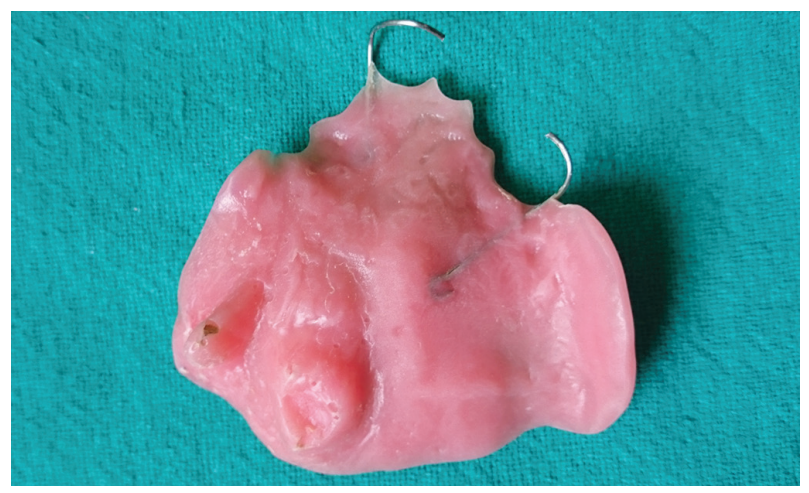

Fig. 2: Interim obturator with circumferential clasp on 11 and 14

The Netherlands) - were inserted at the region of maxillary left central and lateral, and healing screws were placed (Fig. 3). The existing obturator was relined with temporary soft liner (Super Soft, GC America, Alsip, Illinois, USA). After 6 months of osseointegration, second-stage surgery for exposure of the implant was performed, ball abutment standards (Myriad Plus, Equinox Medical Technology) were screwed, and $20 \mathrm{~N} / \mathrm{m}^{2}$ torque was given on both the implants. Maxillary right canine and first premolar were prepared for receiving male attachments of posts number 520-02 (AccessPost Overdenture, EDS, $S$ Hackensack, New Jersey, USA). Trial insertion of the post was done to determine the fit followed by postcementation with self-adhesive resin cement (Relyx U200, 3M ESPE, St. Paul, Minnesota, USA).

Maxillary putty wash impression (Affinis, Coltene/ Whaledent, Inc., Mahwah, New Jersey , USA) was made with the plastic transfer cap snugly fitting the ball heads of the implants. The laboratory implant analog was repositioned into the transfer cap followed by a pour of die stone to produce the definitive cast (Fig. 4).

The obturator was fabricated using heat cure acrylic. Then, the female nylon cap attachments were placed on the balls of the overdenture posts. Also, the female

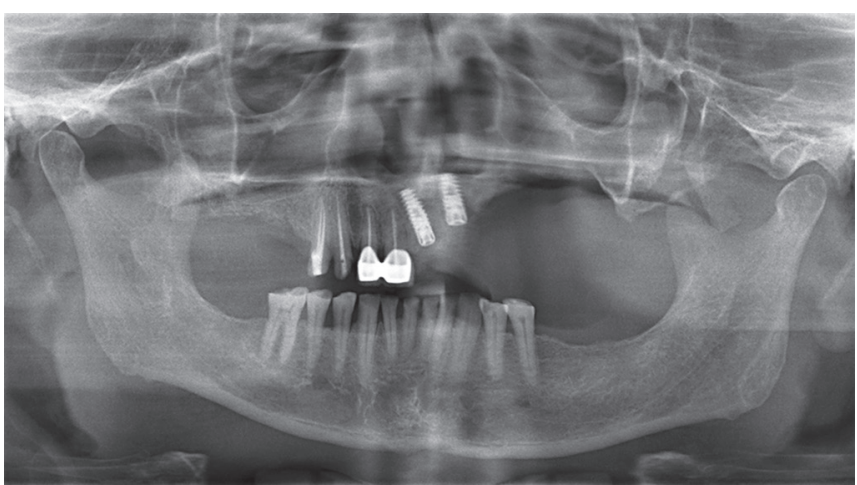

Fig. 3: Panoramic radiograph after implant inserted at the region of 21 and 22

metal housing attachments with the $\mathrm{O}$ ring matrices were pressed over ball abutments. Marking paste was painted on the denture base intaglio surface, and obturator was inserted and relieved to create space for the caps and housings. Relining was done in the relieve area using cold cure acrylic (DPI-RR Cold Cure, Dental Products of India Ltd.) to pick up these female attachments (Fig. 5). The obturator was delivered to the patient after finishing and polishing (Fig. 6). The occlusion and the fit were checked and refined at the follow-up stage.

\section{DISCUSSION}

The rehabilitation of partial and total maxillectomy patients can be done by surgical, prosthetic, or combination of these two approaches depending upon the functional and esthetic requirements of the patient. Even though numerous advances in surgical procedures have been reported, the reconstruction of maxillectomy defects surgically is not always possible because of the general health status of the patient, defect size, and possibility of tumor recurrence. ${ }^{6}$ In such cases, nonsurgical prosthetic rehabilitation is usually advised to the patient.

The size of the defect, the quality and quantity of tissue surrounding the cavity, and the level of direct and 


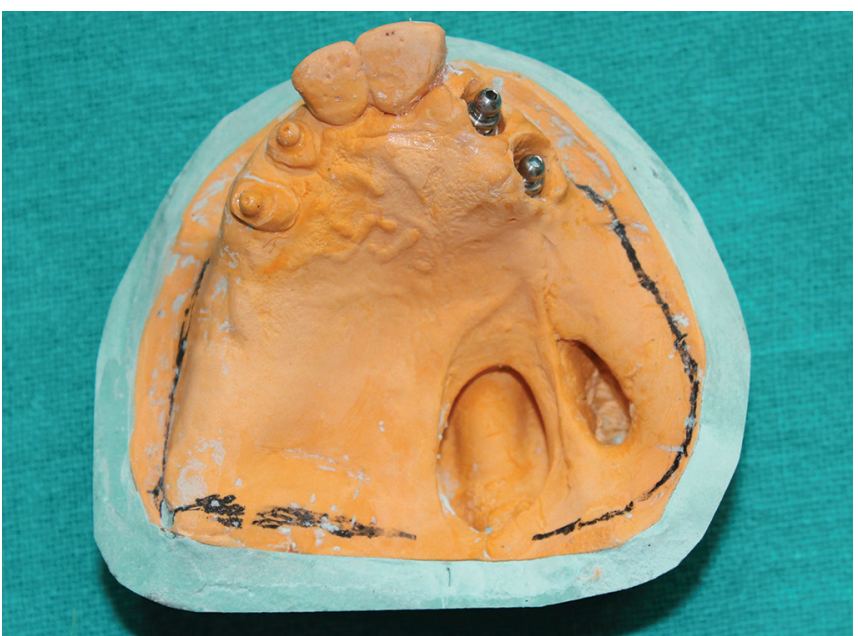

Fig. 4: Definitive cast with implant analog in relation to 21 and 22 and ball socket male attachments in relation to $13,14,21$, and 22

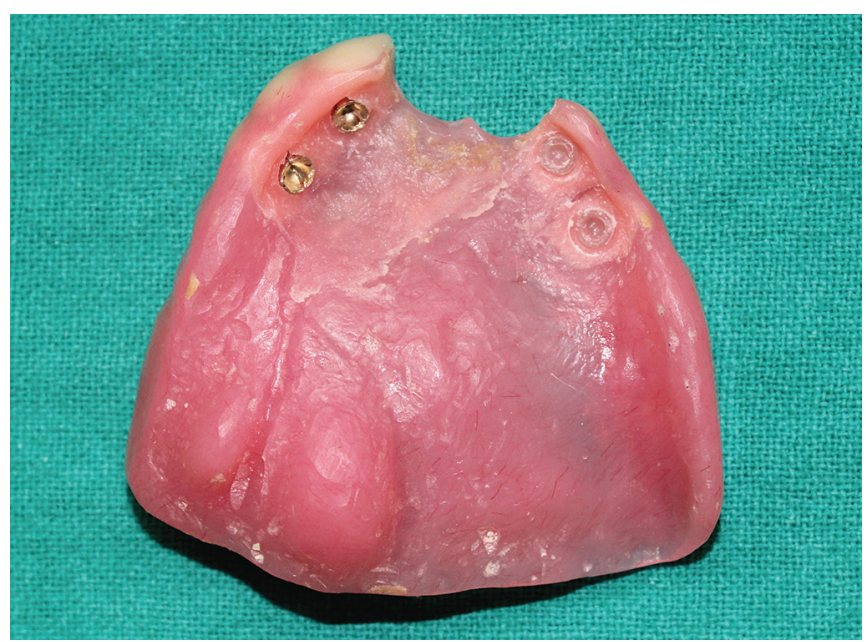

Fig. 5: Tooth implant-supported definitive obturator showing female attachments in relation to $13,14,21$, and 22
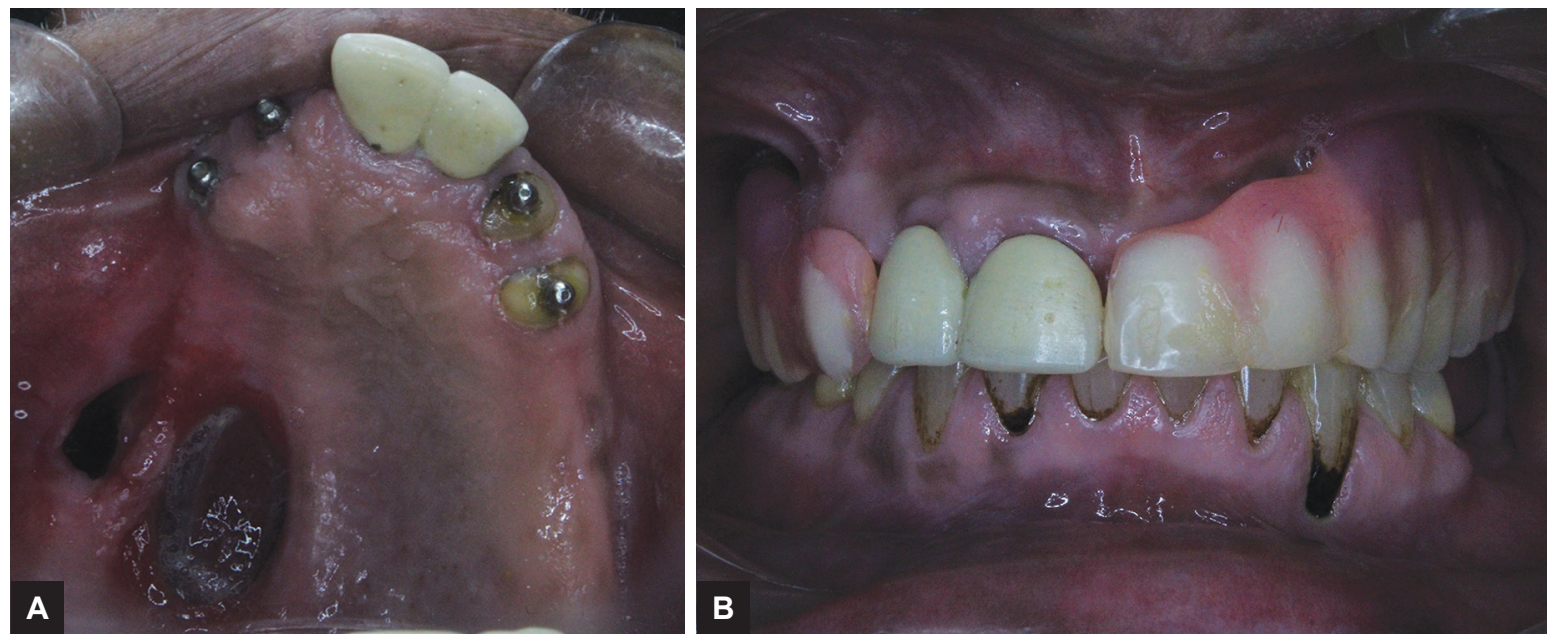

Figs 6A and B: Posttreatment. (A) maxillary intraoral occlusal photograph and (B) frontal intraoral view photograph

indirect retention achieved by the remaining teeth and muscular control are the factors that affect the retention of the obturator. ${ }^{7}$ The supplemental use of osseointegrated implants in these patients may further increase the longevity of the remaining dentition especially those teeth adjacent to the surgical defect. ${ }^{4}$

The implants were inserted nonparallel to each other according to availability of the bone at the placement site. Therefore, ball-socket type of implant and postattachments were selected for supporting obturator as there is no need for parallelism of these attachments, and direct processing of these attachments into the overdentures was possible. Ball and socket attachments help to reduce hydraulic resistance to coupling and lowers functional stress on the implants. ${ }^{8}$ AccessPost Overdenture has thick wall, hollow tube design that demonstrates the strength of a solid shank post. ${ }^{9}$ Additionally, the flange and second tier dissipate functional stresses and prevent bottoming out.

The tooth- and implant-supported obturator is esthetically and functionally acceptable to the patient.
Mastication, swallowing, and speech were significantly improved. The patient is periodically monitored, and the long-term prognosis of this prosthesis looks fair. The overall survival rate for implants supporting maxillofacial prosthesis was reported to be more than $95 \%{ }^{10}$

\section{REFERENCES}

1. The Glossary of Prosthodontic Terms. J Prosthet Dent 2005 Jul;94(1):10-92.

2. Filiz K. Obturator prostheses for hemimaxillectomy patients. J Oral Rehabil 2001 Sep;28(9):821-829.

3. Ueda M, Hibino Y, Niimi A. Usefulness of dental implants in maxillofacial reconstruction. J Long Term Eff Med Implants 1999;9(4):349-366.

4. Murat S, Gurbuz A, Isayev A, Dokmez B, Cetin U. Enhanced retention of a maxillofacial prosthetic obturator using precision attachments. Eur J Dent 2012 Apr;6(2):212-217.

5. Aramany AM. Basic principles of obturator design for partially edentulous patients. Part I: classification. J Prosthet Dent 1978 Nov;40(5):554-557.

6. Keyf F. Obturator prostheses for hemimaxillectomy patients. J Oral Rehabil 2001 Sep;28(9):821-829. 
7. Alqutaibi AY. Enhancing retention of maxillary obturators using dental implants. Int J Contemp Dent Med Rev 2015;010915:1-5.

8. Banton B, Henry MD. Overdenture retention and stabilization with ball-and-socket attachments: principles and technique. J Dent Technol 1997 Sep;14(7):14-20.
9. Cohen BI, Deutsch AS, Musikant BL. Cyclic fatigue testing of six endodontic post systems. J Prosthodont 1993 Mar;2(1): 28-32.

10. Phoenix, RD.; Cagna, DR.; DeFreest, CF. Stewart's clinical removable partial prosthodontics. Hanover Park (IL): Quintessence Publishing; 2008. 